\title{
Erratum to: Towards a Society with Social Protection for All
}

\section{Erratum to:}

H. Zhou, Towards a Society with Social Protection for All, China Insights, https://doi.org/10.1007/978-981-10-4669-8

In the original version of the book, author name "Jun Zhang" and the corresponding affiliation have to be included in book cover and spine, BookFrontmatter, book content as well as the book metadata. The erratum book has been updated with the changes. 